\title{
Acoustic emission during fatigue of Ti-6Al-4V: incipient fatigue crack detection limits and generalized data analysis methodology
}

\author{
D. H. KOHN*,P. DUCHEYNE \\ Department of Bioengineering, University of Pennsylvania, Philadelphia, PA 19104, USA \\ J. AWERBUCH \\ Department of Mechanical Engineering and Mechanics, Drexel University, Philadelphia, PA \\ 19104, USA
}

The fundamentals associated with acoustic emission monitoring of fatigue crack initiation and propagation of Ti-6 Al-4V were studied. Acoustic emission can detect and locate incipient fatigue crack extensions of approximately $10 \mu \mathrm{m}$. The technique therefore can serve as a sensitive warning to material failure. There are three distinct stages during which acoustic emission is generated. These stages are: crack initiation, slow crack propagation and rapid crack propagation. The distinction between the stages is based primarily on the rate of acoustic emission event accumulation. These three stages of acoustic emission correspond to the three stages of the failure process that occurs during fatigue loading. That is, changes in acoustic emission event rate correspond to changes in crack extension rate. Acoustic emission event intensities are greater during crack initiation than during slow crack propagation and greatest during rapid crack propagation. In a given fatigue cycle, event intensities increase with increasing stress and most high-intensity events occur near or at the maximum stress. Acoustic emission may therefore be used with confidence to detect, monitor and anticipate failure, in real-time.

\section{Introduction}

Fatigue processes may be separated into two stages: crack initiation and crack propagation. These stages are often controlled by different mechanisms. The optimal microstructure for maximizing crack initiation resistance is often the microstructure that is least resistant to crack propagation. Therefore, differentiating between initiation and propagation allows one to determine whether microstructural changes that improve either initiation or propagation resistance should be used to improve fatigue strength.

Acoustic emission (AE) is suitable for detecting incipient fatigue cracks, differentiating between fatigue crack initiation and propagation, and analysing mechanisms of failure [1-3]. For titanium and its alloys, $\mathrm{AE}$ has been measured during tensile and compressive deformation [4-8], fracture toughness testing [9, 10], fatigue $[2,3,11,12]$ and stress corrosion cracking [3].

In this study, the characteristics of $\mathrm{AE}$ generated due to fatigue cracking of $\mathrm{Ti}-6 \mathrm{Al}-4 \mathrm{~V}$ were investigated. AE techniques were employed to detect incipient fatigue crack formation and to analyse fatigue crack propagation in Ti-6Al-4V. Data determining the degree of sensitivity with which $\mathrm{AE}$ can be used to detect and locate fatigue crack initiation in $\mathrm{Ti}-6 \mathrm{Al}-4 \mathrm{~V}$ are presented. In addition, a generalized AE data analysis methodology which could be used in future studies is also presented. In a companion paper [13], the methodologies presented here are employed to analyse the effect of material variables and failure mechanisms on AE during fatigue loading of $\mathrm{Ti}-6 \mathrm{Al}-4 \mathrm{~V}$.

\section{Methods and materials}

\subsection{Materials and specimens types}

Specimens were obtained from $3.2 \mathrm{~mm}$ thick forgedannealed, extra low interstitial (ELI) Ti-6Al-4V plate material with an equiaxed microstructure. The chemical composition of the as-received material (Table I) conformed to ASTM F-136 [14]. Specimens were machined so that the direction of loading was parallel to the rolling axis of the plate. The dimensions of the specimens are indicated in Fig. 1. Double-edge notched specimens were used to promote fatigue cracking at a specific location.

\subsection{Fatigue testing procedure and $A E$ data acquisition}

Tension-tension fatigue tests $(R=0.1)$ were performed using a closed loop servo-hydraulic mechanical testing machine (Instron Model 1331). Tests were

* Address for correspondance: Department of Biologic and Materials Sciences, School of Dentistry, The University of Michigan, Ann Arbor, MI 48109, USA. 
TABLE I Chemical composition of as-received Ti-6Al-4V

\begin{tabular}{lll}
\hline Element & As-received (wt \%) & ASTM F136-84 (wt \%) \\
\hline $\mathrm{N}_{2}$ & 0.015 & 0.05 (max.) \\
$\mathrm{C}$ & 0.010 & 0.08 (max.) \\
$\mathrm{H}_{2}$ & 0.007 & 0.012 (max.) \\
$\mathrm{Fe}$ & 0.150 & 0.25 (max.) \\
$\mathrm{O}_{2}$ & 0.112 & 0.13 (max.) \\
$\mathrm{Al}$ & 5.90 & $5.50-6.50$ \\
$\mathrm{~V}$ & 3.50 & $3.50-4.50$ \\
$\mathrm{Ti}$ & Balance & Balance \\
\hline
\end{tabular}

Chemical analysis of Ti-6Al-4V supplied by Titanium Industries Central Service Center, Shaumburg, IL.

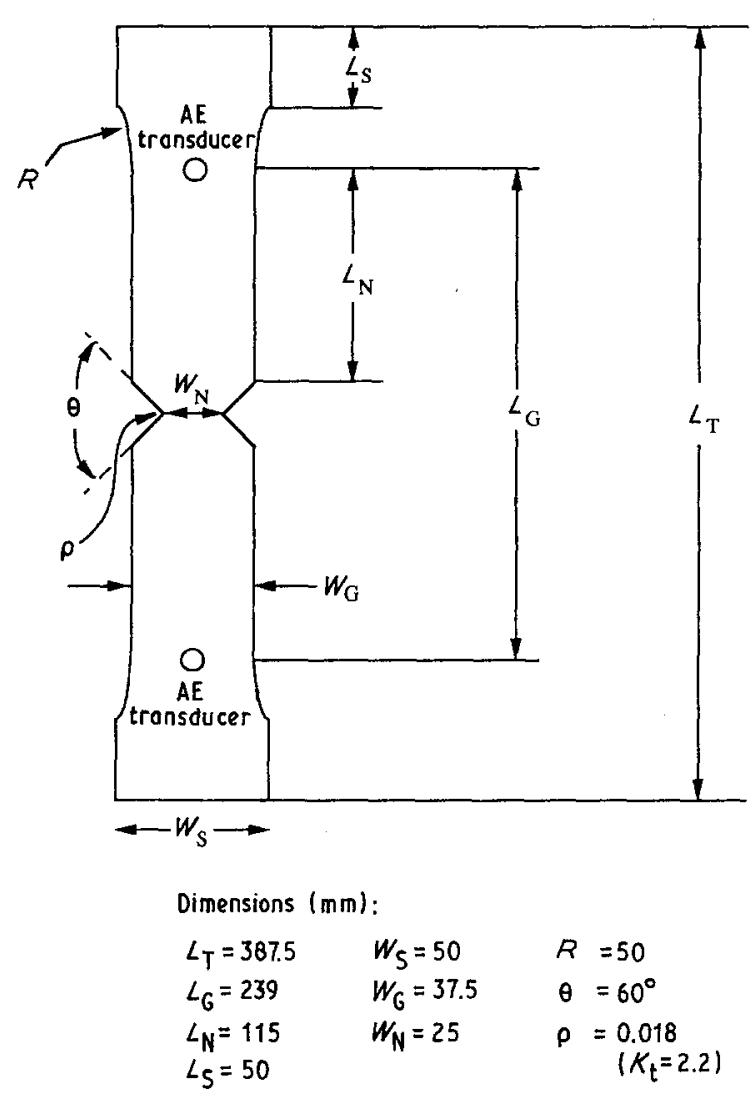

Figure I Schematic illustration of AE specimen design, with dimensions.

conducted under load-controlled mode, with a sinusoidal load cycle, at different frequencies ranging from $0.01-1.0 \mathrm{~Hz}$. For most tests, the maximum stress was $70 \%$ ultimate strength of the material (600 MPa). Prior to the first fatigue cycle, the specimens were statically loaded (under stroke-controlled mode, at a rate of $0.05 \mathrm{~mm} \mathrm{~min}^{-1}$ ) to determine if there were any flaws in the material which might be sources of AE. The amount of damage accumulation from these flaws during the initial loading was therefore determined. Table II summarizes the different tests performed.

Two AE sensors were mounted, equidistant from the notch, on to the two neck regions of the specimens (Fig. 1). After cleaning the specimens with acetone, the sensors were coupled to the specimens with a water soluble acoustic couplant (Dunegan/Endevco AC-WS) and fixed in place by using duct tape.

AE was monitored with Physical Acoustics Corporation (PAC) 3000/3004 instrumentation. The
TABLE II AE testing matrix: $S=\sigma_{\max } / \sigma_{\mathrm{ult}} ; f$ is the loading frequency

\begin{tabular}{lll}
\hline Specimen no. & $S$ & $f(\mathrm{~Hz})$ \\
\hline EA-1 & 0.8 & 0.01 \\
EA-2 & 0.7 & 0.01 \\
EA-3 & 0.7 & 0.01 \\
EA-4 & 0.6 & 1.0 \\
EA-5 & 0.5 & 1.0 \\
\hline
\end{tabular}

TABLE III AE Instrumentation and operating parameters

\begin{tabular}{ll}
\hline Transducers: & PZ Type R-15 \\
& $150 \mathrm{kHz}$ resonance frequency \\
Pre-amplifier: & Model $1220 \mathrm{~A}$ \\
& $40 \mathrm{~dB}$ fixed gain \\
& $100-300 \mathrm{kHz}$ filter \\
& $40 \mathrm{~dB}$ gain \\
Post-amplifier: & $1.0 \mathrm{~V}$ \\
Threshold & $1 \mathrm{~ms}$ \\
Dead time & 0.1 frequency $^{-1} \mathrm{~s}$ \\
Time base & \\
\hline
\end{tabular}

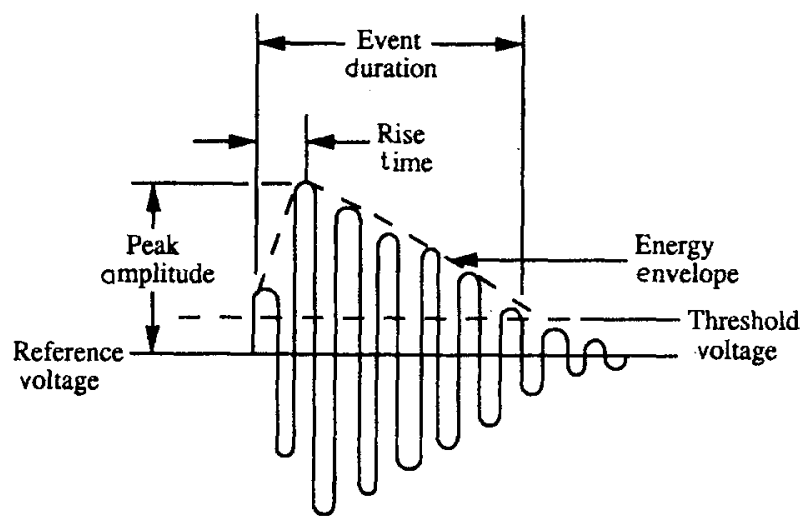

Figure 2 Schematic illustration of AE event waveform and characteristics.

pertinent operating parameters are listed in Table III. AE data were processed with a two-channel AE distribution analyser/locator. Data were collected, stored and analysed using PAC software on a PAC 3000 computer. Results include linear source location, analysis of the number of acoustic waves (AE events) and the intensities of the $\mathrm{AE}$ events. Event intensities are a collective term for event amplitude, number of threshold crossings, defined as counts per event, event duration, event energy counts, and event rise time (Fig. 2). The linear source location provides the site at which AE events were generated. Source location is determined by the $\mathrm{AE}$ system calculating the time difference between the arrival of the same acoustic wave at the two sensors. The smaller the time difference, the closer was the source to the notch tip. A detailed description of the event intensity analyses is given in Section 2.4 .

Monitoring of fatigue crack propagation was also performed optically through a closed circuit television system (CCTV). A video camera (Panasonic Intralux 6000,30 frames $\left.^{-1}\right)$ with a high magnification $(\times 125)$ microscope zoom lens was used to monitor visually 
fatigue crack propagation. The camera was mounted on an electrically controlled, movable platform which had a $25 \mathrm{~mm}$ vertical span and a $50 \mathrm{~mm}$ horizontal span. Thus, the full width and length of the notched region could be visually inspected for surface cracks. Fatigue crack propagation was recorded onto a video cassette recorder for post-test correspondence with the AE data.

\subsection{Correspondence between onset of $A E$ and fatigue crack initiation}

AE sensitivity to incipient fatigue cracking was established by relating the cycle number of the first $\mathrm{AE}$ event to the cycle number of the first evidence of a fatigue crack as observed visually via the CCTV. Crack length measurements were taken from the CCTV when the specimen was under maximum stress. Crack extension was defined as the distance from the previously measured crack tip to the furthest continuous visible point of damage. The total crack length was the sum of the piecewise continuous linear crack segments.

\subsection{AE data analysis techniques}

The following AE parameters were recorded and analysed: number of events, $E$, event amplitude, $A$, counts per event, $C$, event duration, $D$, and event energy counts ( $E N$ counts). Subsets of the recorded events were created, based on event location, fatigue cycle number, and stress range at which the events were generated. Also, AE events were analysed in terms of their intensities, and subsets of event intensities generated within different ranges of location, fatigue cycle number and stress level were established.

Spatial filtering of events was performed to eliminate extraneous emission. Only events generated within the central $60 \%$ of the gauge length were analysed. Additional spatial filtering was performed to analyse only events generated at the crack tip.

Each subset of events was analysed in four ways: (1) average event intensities; (2) cumulative number of events as a function of cumulative number of fatigue cycles ( $E-N$ curves); (3) two-dimensional location distribution histograms $(\mathrm{LDH})$ of events and intensity distribution histograms (IDH) of events and (4) threedimensional LDH and IDH of events. Location and intensity distribution histograms are general terms for the distribution of events and event intensities as functions of location, cycle number and/or applied load. Fig. 3 is a flowchart of the general data analysis methodology employed.

\subsection{Identification of friction emission}

Generally, a significant amount of emission can be generated from grating between existing fracture surfaces. This emission, defined here as "friction emission", is distinct from the emission generated by actual damage progression. Friction emission discrimination

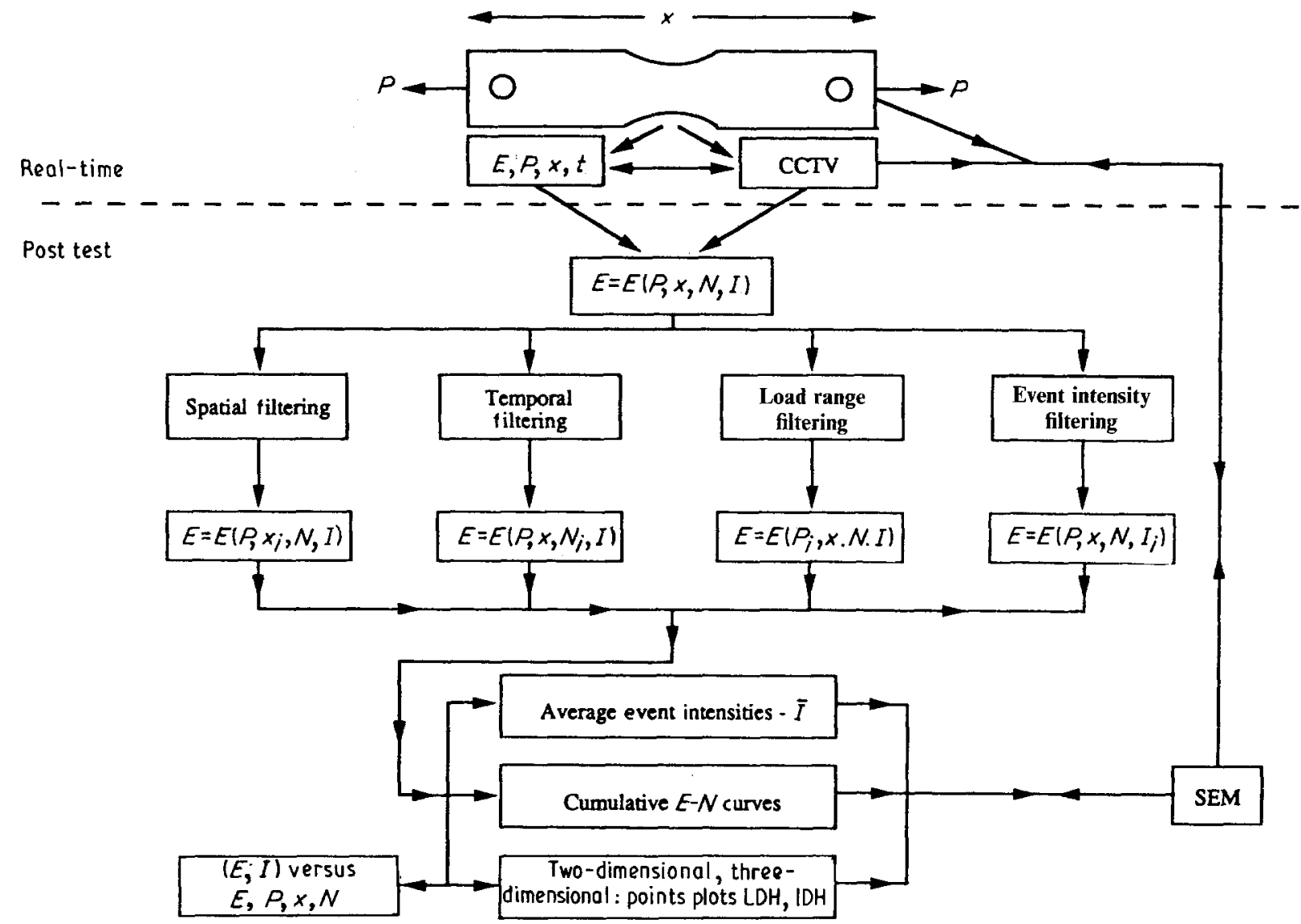

Figure 3 Flowchart of AE data analysis techniques. ( $E=$ AE events, $P=$ load, $x=$ location, $N=$ number of fatigue cycles, $I=$ AE event intensity, $\mathrm{LDH}=$ location distribution histogram of events, $\mathrm{IDH}=$ intensity distribution histogram, of events, CCTV $=$ closed circuit television). 
was based upon analysing specific subsets of events, mentioned above, and also whether events occurred during loading or unloading. For this purpose, the recorded events were separated into two subsets, determined by the stress ranges in which they were generated: events generated between $10 \%$ and $60 \%$ maximum stress and events generated between $95 \%$ and $100 \%$ maximum stress. In this study, it was assumed that at the lower stress range, most of the emission was generated by grating and not by actual damage.

Threshold intensities characteristic of friction emission, referred to as "FRET" (friction emission threshold) values, were identified by screening IDHs of events for low level values of event intensities.

Event intensities representing friction emission were also determined by performing friction verification tests. In these tests, a maximum stress of 0.7 ultimate tensile strength (UTS) was applied until a fatigue crack propagated to a length sufficient to generate emission from both crack extension and crack closure. The maximum stress was then lowered to a level that was insufficient to produce further visual crack propagation (0.2 UTS). All of the AE events generated at the lower stress level were therefore assumed to be due to crack opening and closing only. The intensities of the events recorded during fatigue cycling at the lower maximum stress level determined the FRET values. The fatigue stress amplitudes were alternated several times (Table IV) to determine the average event intensities representing friction emission.

\section{Results and discussion}

\subsection{AE source location and correspondence} with fatigue crack initiation

A curve of cumulative number of events versus the number of fatigue cycles ( $E-N$ curve) for a representative test (EA-2) is shown in Fig. 4a. From this curve, the occurrence of the first $\mathrm{AE}$ event (at cycle number 88 ) is easily seen. At this fatigue cycle number, a fatigue crack approximately $32 \mu \mathrm{m}$ long was optically observed (Fig. 4b). The correspondence between this $\mathrm{AE}$ result and the progression of damage, detected optically, is indicative of $\mathrm{AE}$ sensitivity to fatigue crack initiation. Similar correspondences were made for all other specimens (Table V). Listed in the table are the fatigue cycle numbers at which $\mathrm{AE}$ initiated for

TABLE IV Loading sequence for friction verification test. $S=\sigma_{\max } / \sigma_{\mathrm{ult}} ; f=$ loading frequency

\begin{tabular}{lllc}
\hline Step & $S$ & $f(\mathrm{~Hz})$ & Number of cycles \\
\hline I $^{\mathrm{a}}$ & 0.7 & - & - \\
$2 \mathrm{~A}$ & 0.2 & 0.1 & 100 \\
2B & 0.2 & 1.0 & 1000 \\
3 & 0.5 & 0.1 & 51 \\
4 & 0.2 & 1.0 & 986 \\
5 & 0.5 & 0.1 & 93 \\
6 & 0.1 & 1.0 & 1000 \\
7 & 0.5 & 1.0 & 248 \\
\hline
\end{tabular}

a Crack initiated during initial static loading, therefore loading at 0.7 UTS was skipped, to avoid catastrophic failure.
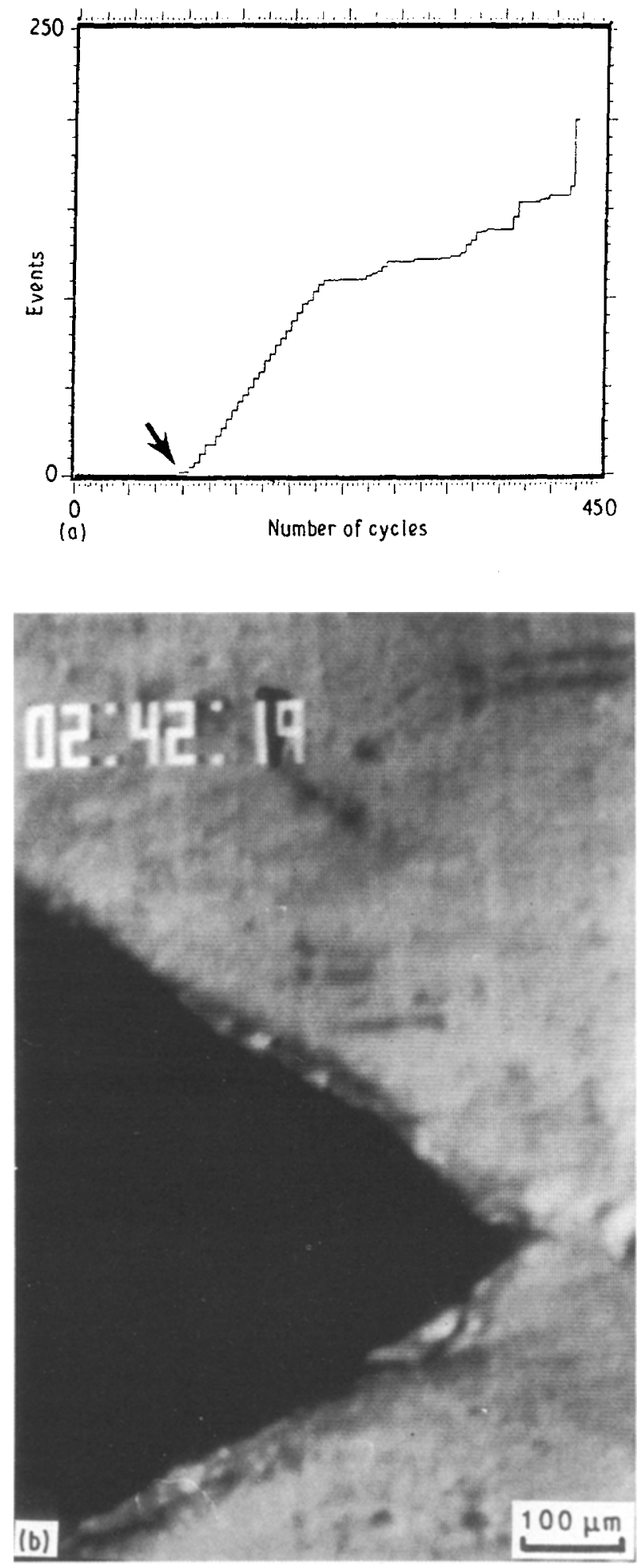

Figure 4 (a) Curve of cumulative number of events versus number of fatigue cycles ( $E-N$ curve) for specimen EA-2, showing the onset of $\mathrm{AE}$ at $N=88$. (b) Photograph showing a crack approximately $32 \mu \mathrm{m}$ long at the notch-tip of specimen EA-2 at $N=88$.

each specimen, the cycle numbers at which fatigue crack initiation was first observed optically, and the corresponding crack lengths.

The smallest crack lengths that could be reliably detected optically were approximately $10 \mu \mathrm{m}$. In all specimens tested, emission initiated prior to, or at the same time as the optical detection of fatigue crack initiation. It should be noted that optical observations can reveal only surface damage, while emission is generated throughout the entire thickness of the specimen. Therefore, it can be concluded that the AE 
TABLE V Summary of AE and CCTV sensitivity for fatigue tests

\begin{tabular}{llllllllr}
\hline Specimen & $\sigma_{\max } / \sigma_{\mathrm{UTS}}$ & $\sigma_{\max }(\mathrm{MPa})$ & $f(\mathrm{~Hz})$ & $\begin{array}{l}\text { Cycle of } \\
\text { 1st AE event }\end{array}$ & $\begin{array}{l}\text { Cycle of } \\
\text { 1st optical } \\
\text { observation }\end{array}$ & $\begin{array}{l}\text { Crack length }(\mu \mathrm{m}) \\
\text { at 1st optical } \\
\text { observation }\end{array}$ & $\begin{array}{l}\text { Cycles to } \\
\text { failure }\end{array}$ & $\begin{array}{l}\text { Total events } \\
\text { at failure }\end{array}$ \\
\hline EA-1 & 0.8 & 717 & 0.01 & 10 & 13 & 10 & 14 & 11 \\
EA-2 & 0.7 & 607 & 0.01 & 88 & 88 & 32 & 415 & 200 \\
EA-3 & 0.7 & 607 & 0.01 & 8 & 102 & 56 & $175^{\mathrm{a}}$ & 267 \\
EA-4 & 0.6 & 530 & 1.0 & 74 & 133 & 40 & 171 & 40 \\
EA-5 & 0.5 & 453 & 1.0 & 2 & 24 & 10 & 790 & 125 \\
\hline
\end{tabular}

${ }^{a}$ Specimen not tested to failure.

technique can serve as a sensitive early warning method for detecting incipient fatigue cracks even smaller than $10 \mu \mathrm{m}$ long. In other words, based on the data in Table V, the AE technique is more reliable to monitor fatigue cracking than conventional techniques, which use optical observations.

\subsection{AE data analysis methodology}

The $E-N$ curves, such as the one shown in Fig. 5a, are defined by three separate regions: (I) early emission, indicative of fatigue crack initiation; (II) a relatively stable crack propagation phase, as indicated by the
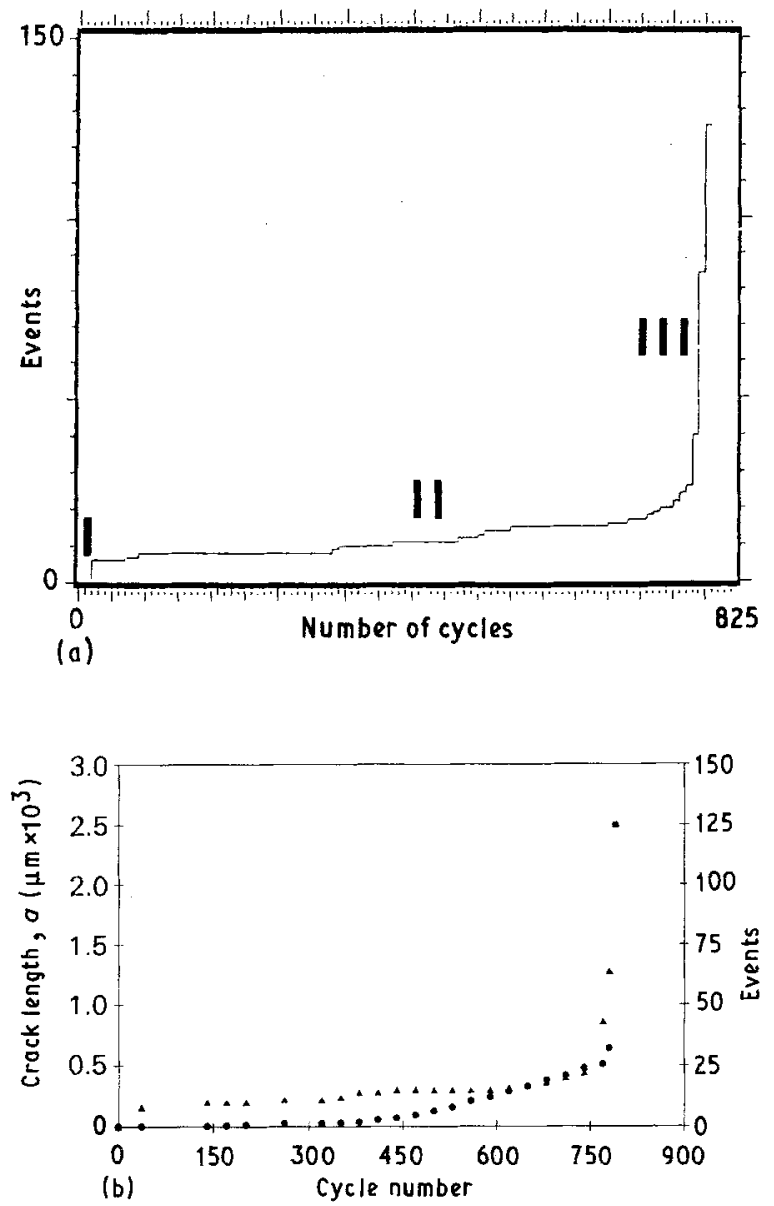

Figure 5 (a) Curve of cumulative number of events versus number of fatigue cycles for specimen EA-5, showing three stages at which AE is generated: crack initiation (I), slow crack propagation (II) and rapid crack propagation (III), (b) Superposition of curves of ( $\mathbf{A}$ ) cumulative number of events versus cumulative number of fatigue cycles and (O) cumulative crack length versus cumulative number of fatigue cycles for specimen EA-5. lower emission rate; and (III) an increase in the amount and rate of emission as unstable crack propagation and catastrophic fracture occur. The onset of unstable crack propagation is seen from the rapid increase in the event rate at fatigue cycle number 770 . The increase in emission rate coincided with an increase in the rate of the actual crack extension, as observed optically.

Changes in the rate of emission followed changes in the rate of crack extension. This result is evident when a comparison is made between the measurement of crack length as a function of the number of fatigue cycles ( $a-N$ curves) and the $E-N$ curves (Fig. 5b). Measurements of the fracture surface in the SEM showed that the length of the fatigue region of this specimen was approximately $6 \mathrm{~mm}$. In comparison, the crack length measured with the CCTV just prior to catastrophic failure was $6.4 \mathrm{~mm}$. Thus, it can be concluded that in addition to detecting incipient fatigue cracks of less than $10 \mu \mathrm{m}, \mathrm{AE}$ also offers the ability to detect and anticipate fatigue crack propagation accurately and provide early warning to catastrophic fracture.

By recording the source location of all AE events, a location distribution histogram (LDH) of events can be plotted. This method of representing the data provides a more graphical documentation of the increased AE activity. It thereby reveals the location of material damage accumulation and enables fracture sites to be anticipated in real-time. The LDH of all events accumulated during the fatigue life of sample EA-2 (Fig. 6) shows that, initially, most of the emission was generated at the notch tip (i.e. location 50). As the fatigue crack grew, additional emission was generated from other locations along the specimen length, indicating failure progression. It should be noted that the few events generated away from the notch tip during the initial phases of the fatigue cycling may have been caused by surface imperfections.

\subsection{Friction emission discrimination methods}

The repeated grating between existing fracture surfaces during long portions of the fatigue cycling can generate emission repeatedly at a given material point (i.c. at the same location and at the same stress level). Grating can occur when crack faces come into contact during the unloading phase of the load cycle or, due to the ruggedness of the fracture surfaces (Fig. 7), when crack faces slide over one another during reloading. 


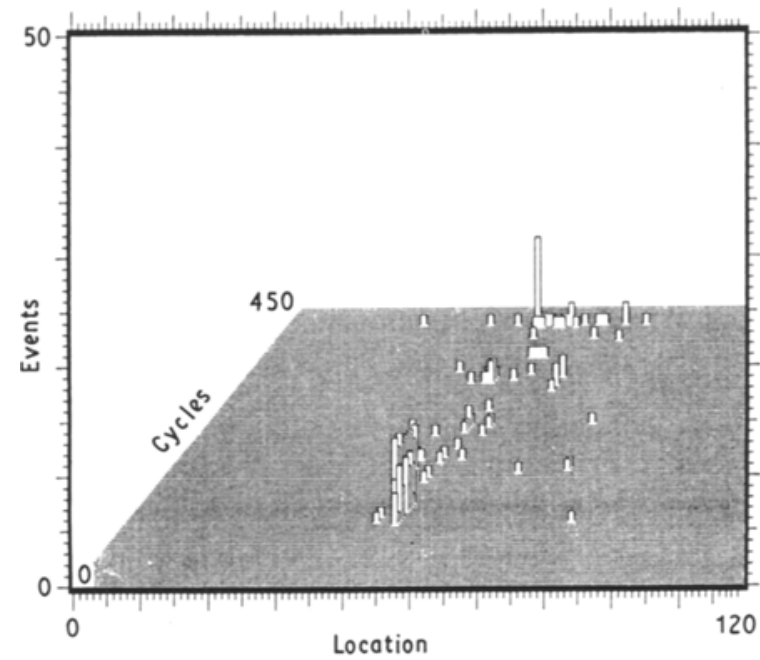

Figure 6 Location distribution histogram (LDH) for specimen $\mathrm{EA}-2\left(\sigma_{\max }=607 \mathrm{MPa}, E=200\right.$ events).

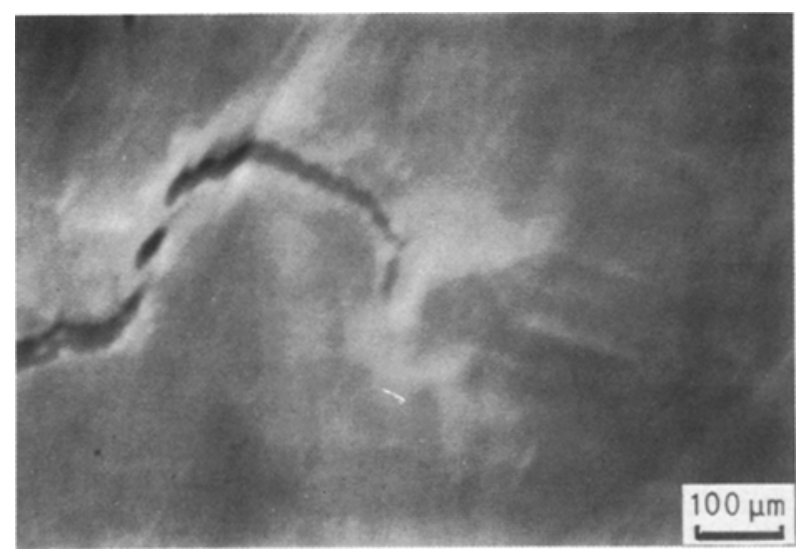

Figure 7 Photograph of sliding between crack surfaces and the potential for friction emission.

In contrast, material damage and the resultant AE are singular. In other words, a wave is generated only once, at the time the damage is created in that specific volume of material. The data presented in Figs 8 and 9, further support the occurrence of friction emission. Analysis of these data outlines how AE caused by grating can be distinguished from AE caused by damage progression.

Many events occurred at lower stresses and were of lower intensities (Fig. 8). The distribution of event intensities generated during a representative test (Fig. 5b) are shown as functions of the number of fatigue cycles (Fig. 8a-d) and stress (Fig. 8e-h). These distributions are referred to as "point plots". Each point in a "point plot" indicates that an event of a given intensity occurred at a given fatigue cycle number or stress level, respectively.

It is interesting to note that most events are generated at the low stress ranges, indicating that $\mathrm{AE}$ occurs primarily due to grating among existing fracture surfaces. In order to illustrate this point, events are separated into three subsets: (1) the total number of events generated; (2) events generated within $10 \%-60 \%$ maximum stress; and (3) events generated within $95 \%-100 \%$ maximum stress. The $E-N$ curves for events generated in these stress ranges (Fig. 9), are representative of all the specimens tested. These curves clearly indicate that, most often, events were generated in the lower $60 \%$ of the stress cycle and they accumulated continuously. Although the emission generated at low stresses is more continuous than the emission generated at high stresses, it is clear from the $E-N$ curves that events generated in the low stress range also have an intermittent nature. The reason for the intermittent nature is that as a fatigue crack propagates, the notch-tip geometry changes, resulting in changes in the nature and amount of grating.

Events that were generated at the low stress range were accumulated during consecutive cycles of the fatigue loading, whereas the events generated at the higher stress range occurred more intermittently (Fig. 9). These more continuous event accumulations correspond to the continuous grating among existing fracture surfaces. The more intermittent events, generated at the higher stresses, correspond to sudden crack growth. A further indication of friction is that events were generated at progressively lower stresses with increasing fatigue cycle number (Fig. 10). The reason for this trend in the AE data is because as a crack grows, a lower load is required to cause the crack faces to come into contact with each other.

Friction emission is generated provided a crack has propagated to a sufficient length. This fact explains the finding that, in a given test, initially, most events were generated at the high stress range and, as the fatigue loading progressed, more events were generated at the lower stress range. For example, for the specimen whose $E-N$ curve is shown in Fig. 5a, low stress events occurred only after 150 fatigue cycles, at which stage the fatigue crack was approximately $100 \mu \mathrm{m}$ long. This interpretation of the AE data is supported by work on short crack growth in titanium alloys, which showed that crack closure effects are negligible for short $(a<100 \mu \mathrm{m})$ cracks $[15,16]$.

Although material damage and friction both release energy, it should be expected that emission produced by the formation of new material surfaces releases more energy than fretting among existing fracture surfaces. Therefore, friction emission should be characterized by low level event intensities, which occur throughout the entire range of cyclic stresses. The intensity distributions (Fig. 8) show that most of the event intensities were below certain threshold values. These friction emission threshold (FRET) intensities, are: $A=50 \mathrm{~dB}, E N=20$ counts, $C=20$ and $D=120 \mu \mathrm{s}$.

It should be noted that grating can occur at both the lower and upper stress ranges. Therefore, emission generated at the upper stress range cannot necessarily be attributed to damage. In other words, emission caused by damage cannot be identified by simply differentiating events based on the stresses at which they were generated. Consequently, the friction emission which occurs at the upper stress range is identified by its event intensities.

The data shown in Table VI illustrate the above argument. A specimen was subjected to the fatigue loading sequence outlined in Table IV. Prior to the 


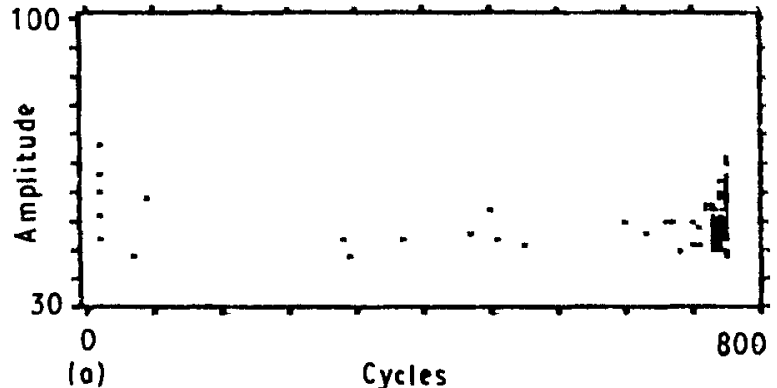

(a)

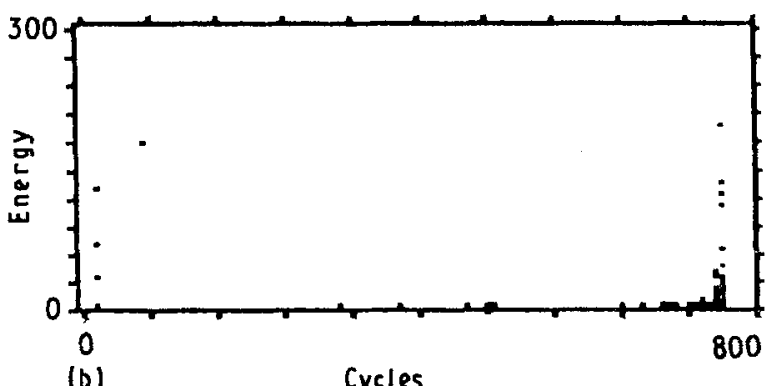

(b)
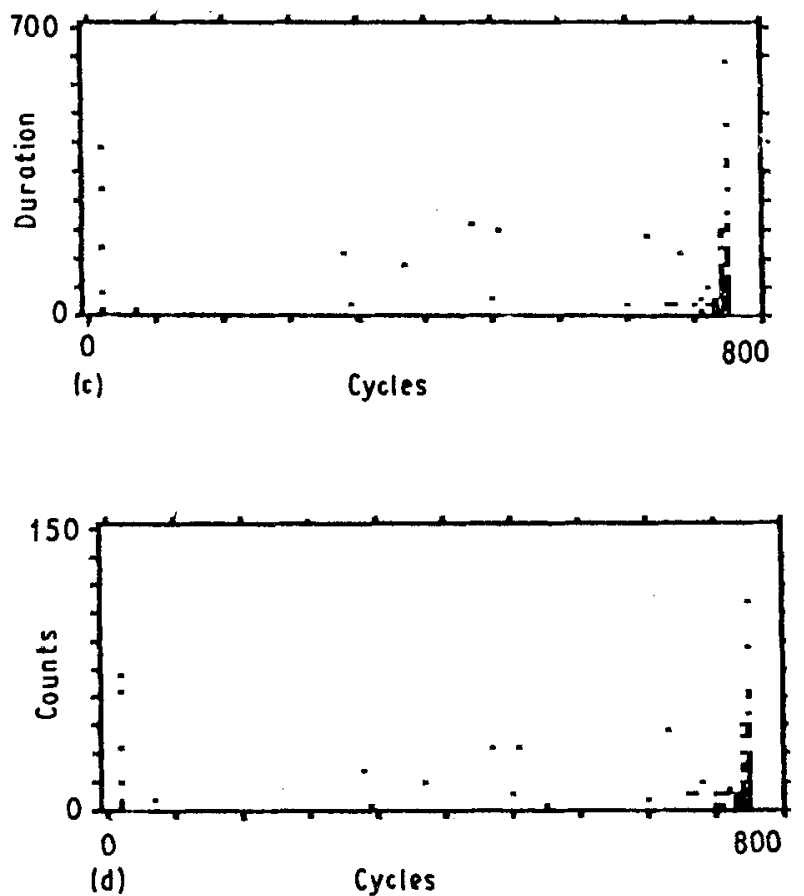
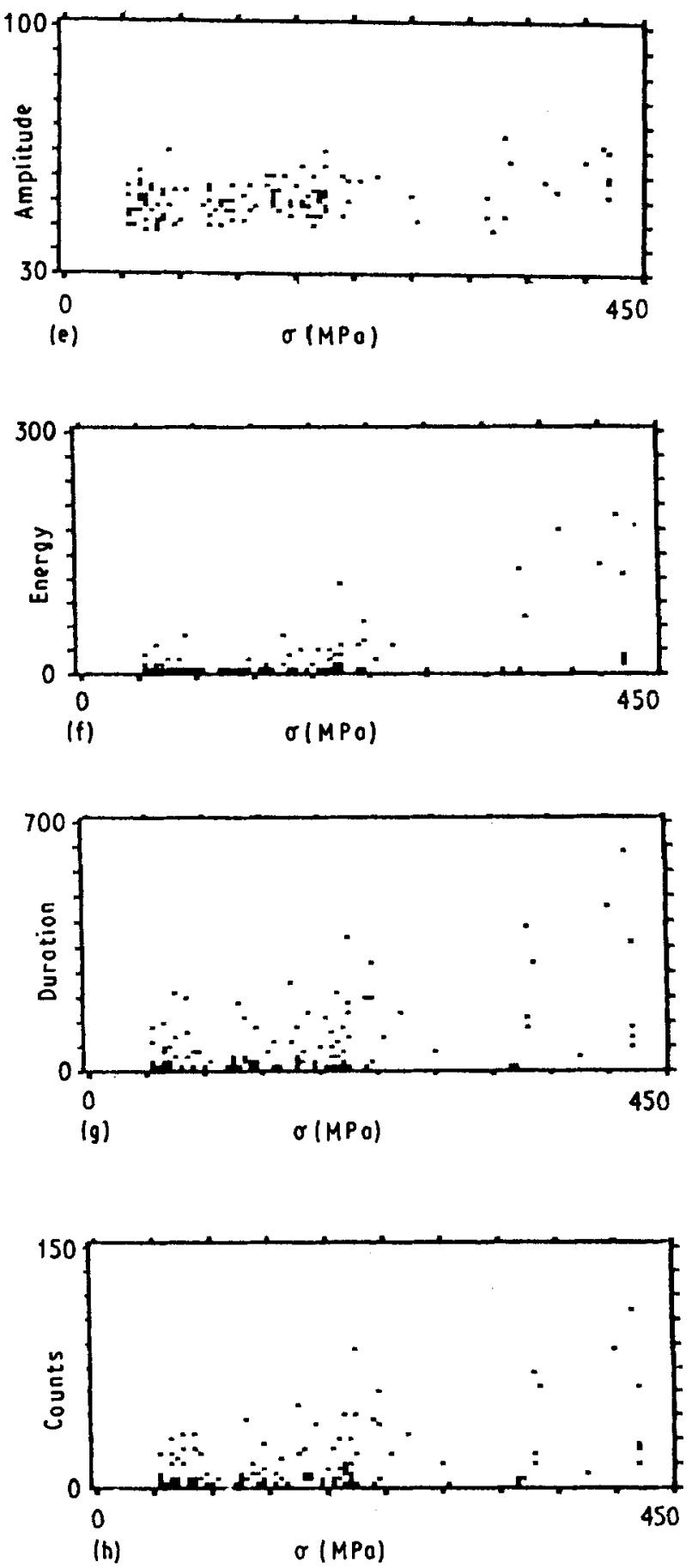

Figure 8 "Point plots" of (a-d) event intensities versus fatigue cycles, and $(\mathrm{e}-\mathrm{h})$ event intensities versus stress for specimen EA-5 $\left(\sigma_{\max }=450 \mathrm{MPa}, \dot{N}=790, E=125\right.$ events $)$.

fatigue loading, the specimen was loaded quasi-statically to $70 \%$ ultimate strength, causing a crack of approximately $3.8 \mathrm{~mm}$. During loading steps $2 \mathrm{~A}, 2 \mathrm{~B}, 4$ and 6, no new damage is expected to occur, and in fact, no detectable crack propagation was optically observed during these steps. Yet, a large number of events was generated. These events are attributed to the grating among the fracture surfaces of the existing crack $(a=3.8,3.9,5.2 \mathrm{~mm})$ at the beginning of steps 2 , 4 and 6 , respectively. The average intensities of these events were all relatively low (Table VI). In contrast, the events generated in steps 3,5 , and 7 (Table VI), during which the specimen was loaded to a higher maximum stress, were all of higher intensities. The average intensities attributed to grating were derived from the intensities of the events generated in the lowload steps, and determined to be: $A=51 \mathrm{~dB}, E N=6$ counts, $C=6$ and $D=29 \mu$ s.

The fact that event intensities increased with increasing stress range has therefore been demonstrated in two ways: by analysing the intensity distributions (Fig. 8) and by alternating the maximum stresses applied (Table VI). It should be noted that the FRET values differ slightly, depending on which method was used to determine these values. Analysis of the intensity distributions yields threshold values, or the maximum event intensities attributed to grating. On the other hand, alternating the maximum stresses yields average intensities accumulated over the course of the loading cycle, and these values are therefore lower. It 


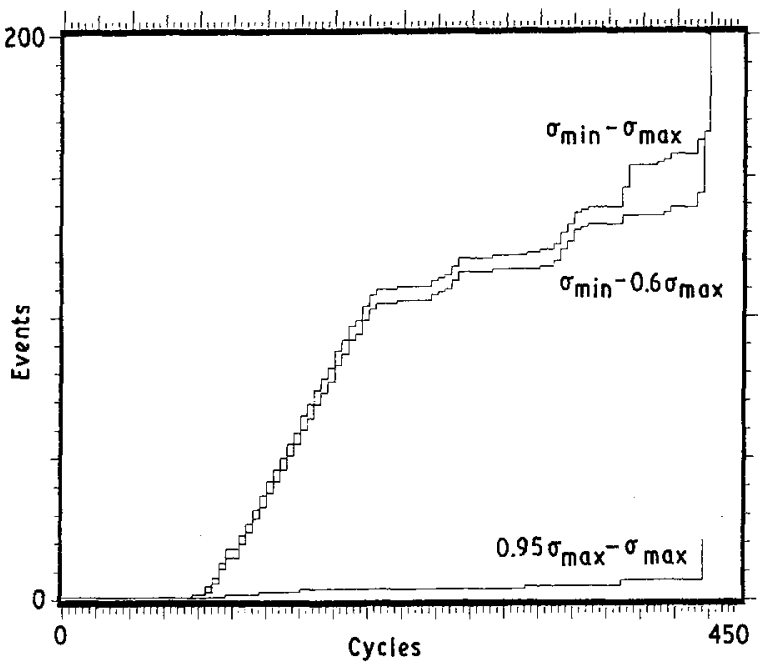

Figure 9 Curves of cumulative number of events versus cumulative number of fatigue cycles for specimen EA-2, showing events generated in different stress ranges.

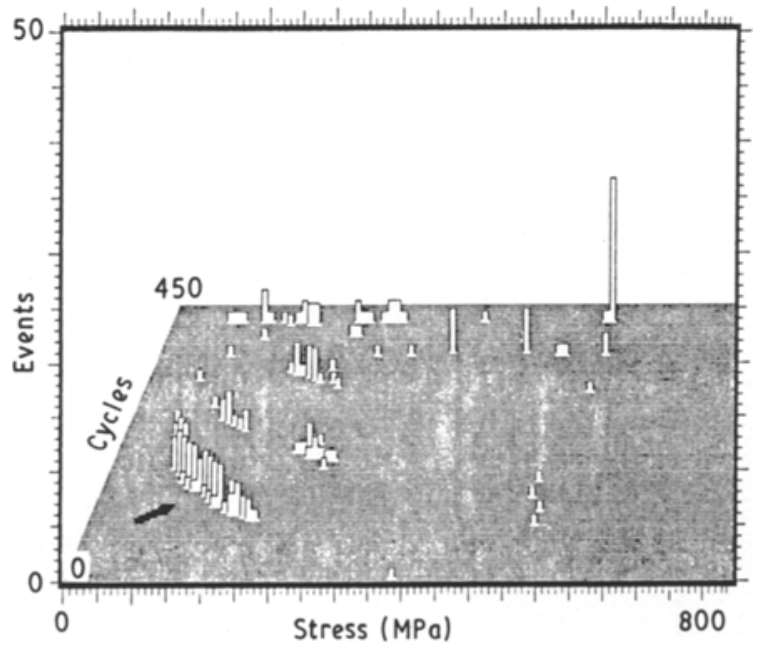

Figure 10 Three-dimensional plot of generated events as a function of stress and fatigue cycle number for specimen EA-2 $\left(\sigma_{\max }=607 \mathrm{MPa}, E=200\right.$ events).

should also be noted that, in general, FRET values may change with the quality of the fracture surface, applied load and loading frequency.

The three-dimensional plots shown in Fig. 11 reveal that for the events generated during step 3 (Table VII), emission occurred at specific locations and stresses, during specific fatigue cycle ranges. Because this emis-

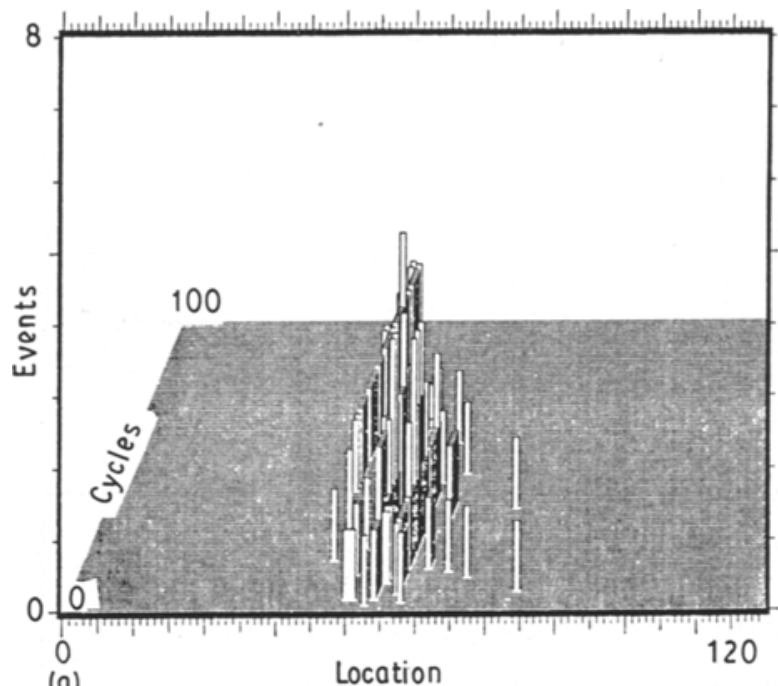

(a)

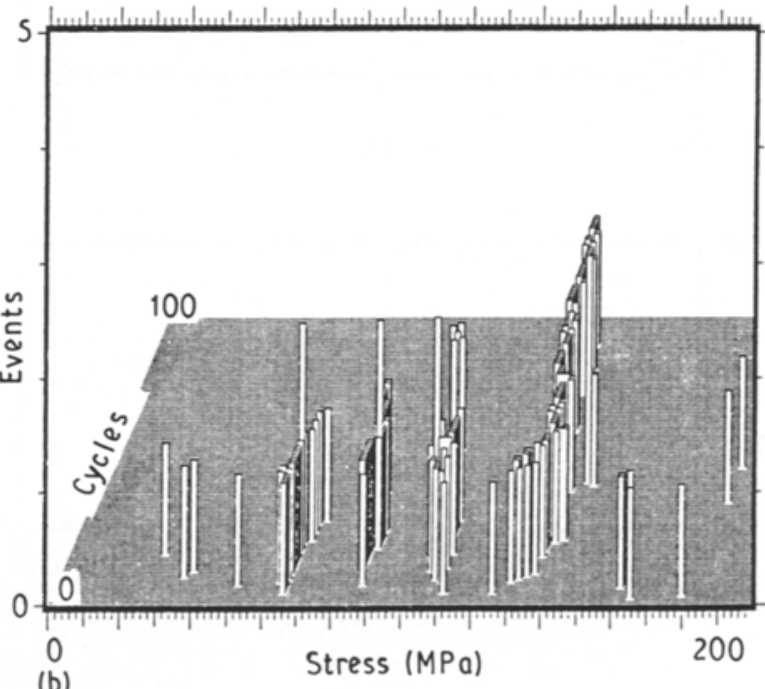

Figure 11 Friction verification plots from low-loading step of FRET test: three-dimensional plots of generated events as a function of (a) location and fatigue cycle number and (b) stress and fatigue cycle number $\left(\sigma_{\max }=207 \mathrm{MPa}, N=100, E=100\right.$ events).

sion occurred repeatedly at specific fatigue cycles, at the same location, same (low) stress and was characterized by the same (low) intensities, it must have been caused by friction. These plots are therefore referred to as "friction verification plots".

Another important consideration with respect to differentiating between damage and friction emission is that in a given fatigue cycle, events due to material damage may be generated to non-peak stresses. The $\mathrm{AE}$ results shown in Fig. 12 indicate that under certain

TABLE VI Results of friction verification test: average event intensities as a function of stress and frequency

\begin{tabular}{lllcccccc}
\hline Step & Max. stress (MPa) & $f(\mathrm{~Hz})$ & Cycles & Events & $\begin{array}{l}\text { Amplitude } \\
(\mathrm{dB})\end{array}$ & Counts & $\begin{array}{l}\text { Duration } \\
(\mu \mathrm{s})\end{array}$ & $E N$ (counts) \\
\hline $1^{\mathrm{a}}$ & - & - & - & - & - & - & - & - \\
2A & 0.2UTS (207) & 0.1 & 100 & 188 & 49 & 8 & 36 & 8 \\
2B & 0.2UTS (207) & 1.0 & 1000 & 934 & 49 & 4 & 16 & 3 \\
3 & 0.5UTS (517) & 0.1 & 51 & 735 & 56 & 30 & 180 & 53 \\
4 & 0.2UTS (207) & 1.0 & 986 & 279 & 55 & 7 & 36 & 7 \\
5 & 0.5UTS (517) & 0.1 & 93 & 489 & 57 & 27 & 146 & 46 \\
6 & 0.1UTS (104) & 1.0 & 1000 & 3 & - & - & - & - \\
7 & 0.5UTS (517) & 1.0 & 248 & 2687 & 56 & 34 & 199 & 63 \\
\hline
\end{tabular}

${ }^{\text {a }}$ Crack initiated during initial static loading, therefore loading at 0.7 UTS was skipped, to avoid catastrophic failure. 


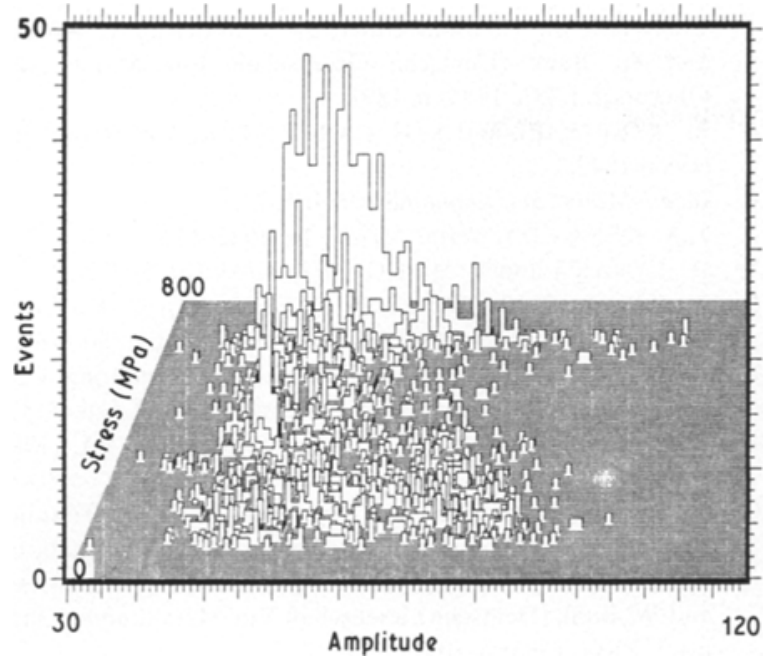

Figure 12 Three-dimensional plot of events generated as a function of stress and event amplitude. Many events were generated at lower stresses. $\left(\sigma_{\max }=730 \mathrm{MPa}, N=6492, E=2026\right.$ events $)$.

loading conditions, high-intensity events may also be generated at the lower stress range. In other words, event intensities greater than the FRET intensities are generated. These events are not necessarily caused by the grating, but rather by plastic flow. It has been shown that the plastic zone growth which precedes crack propagation can occur at stresses lower than the peak stress [17]. Additionally, as demonstrated above, friction emission can be generated at the upper stress ranges. Therefore, provided the maximum stress is sufficient to cause crack propagation, both damage and friction emission may occur at all stress levels. Analysis of these events and determination of FRET values should therefore be based on event intensity levels as well as stress levels, and not just stress levels, as is traditionally done.

\subsection{Analysis of AE from material damage}

Generally, events generated during crack initiation are of higher maximum intensities $(A=70 \mathrm{~dB}$, $E N=180$ counts, $C=75, D=400 \mu \mathrm{s})$ than those events generated during stable crack propagation $(A=54 \mathrm{~dB}, E N=10$ counts, $C=40, D=240 \mu \mathrm{s})$. This is evident from analysing the intensity distributions (Fig. 8). Based on the point plots shown in Fig. 8(a-d), most of the events were generated during final fracture. In addition, the maximum intensities of the events generated during final fracture $(A=72 \mathrm{~dB}$, $E N=202$ counts, $C=113, D=629 \mu$ s) were higher than the intensities of the events generated during stable crack propagation. Moreover, most of these higher intensity events occurred at the higher stresses (Figs $8 \mathrm{e}-\mathrm{h}$ ). However, based on the relative number of "points" in the "point plots" shown in Figs 8e-h, most events occurred at lower stresses. Further analysis of these events (not shown here), indicated that this trend is especially true for those events generated during stable crack propagation.

Continuous crack propagation was observed optically, yet, as indicated in Fig. 9, events generated in the upper stress range occurred intermittently, possibly indicating that $\mathrm{AE}$ sensitivity to crack growth is less than $\mathrm{AE}$ sensitivity to crack nucleation. Events generated at different stress ranges may also be due to different source mechanisms, such as plastic zone extension, crack propagation and friction. The average $\mathrm{AE}$ event intensities are higher at the uppermost stress ranges (Table VII). This is explained by the fact that events generated in the high stress range may mostly be attributed to plastic flow and crack propagation, while events generated in the low stress range may be indicative of grating among fracture surfaces. It has been shown, above, that events generated from crack face grating are of lower intensity than events generated from damage extension.

In summary, the three stages of fatigue crack progression (crack initiation, stable crack propagation and rapid crack propagation) are readily detected and analysed by AE analysis. However, detailed characterization of the $\mathrm{AE}$ events and event intensities indicative of these three stages is only possible if the emission from the grating of the fracture surfaces is characterized first.

\section{Conclusions}

1. AE can detect incipient fatigue crack extensions of $10 \mu \mathrm{m}$, or less, in Ti-6Al-4V; it therefore can serve as a sensitive warning method for analysing material failure.

2. There are three distinct stages during which $\mathrm{AE}$ is generated: crack initiation, slow crack propagation and rapid crack propagation. The distinction between these stages is based primarily on increases in the rate of $\mathrm{AE}$ event accumulation. The rate of $\mathrm{AE}$ event accumulation corresponds to actual crack growth, as observed optically, and can therefore be used to anticipate failure.

3. AE data are interpreted by separating the recorded events into subsets, according to location, ranges of fatigue cycles, stress range and event intensities. Material failure mechanisms follow from the analysis of $\mathrm{AE}$ events in terms of their intensities and variations in intensities with location, fatigue cycle number and stress level.

4. A large number of events were generated by grating. These events are generally of low intensity. Friction emission threshold (FRET) intensity values were determined: $A=50 \mathrm{~dB}, E N=6-20$ counts, $C=6-20$ and $D=30-120 \mu$ s.

TABLE VII Average intensities of AE events generated during different load ranges

\begin{tabular}{lllccc}
\hline Load range $\left(\% \sigma_{\max }\right)$ & Events & Amplitude $(\mathrm{dB})$ & Counts & Duration $(\mu \mathrm{s})$ & $E N($ counts $)$ \\
\hline $0-100$ & 199 & 51 & 12 & 57 & 14 \\
$10-60$ & 166 & 50 & 9 & 37 & 7 \\
$95-100$ & 21 & 57 & 40 & 209 & 62 \\
\hline
\end{tabular}


5. AE event intensities are greater during crack initiation $(A=70 \mathrm{~dB}, E N$ counts $=180, C=75$, $D=400 \mu \mathrm{s}$ ) than during slow crack propagation ( $A=54 \mathrm{~dB}, E N=10$ counts, $C=40, \quad D=240 \mu \mathrm{s}$ ) and are greatest during rapid crack propagation ( $A=72 \mathrm{~dB}, E N=200$ counts, $C=110, D=625 \mu \mathrm{s}$ ). Event intensities also increase as the maximum stress increases, because more material damage is created at higher stresses.

6. By monitoring AE during fatigue loading of $\mathrm{Ti}-6 \mathrm{Al}-4 \mathrm{~V}$, the sequence of failure processes can be easily monitored and catastrophic fracture can be anticipated.

\section{Acknowledgements}

This work was supported by a grant in aid from DePuy, Inc., Warsaw, IN 46580. The assistance of Shahrokh Ghaffari in performing the experiments and analysing the data is greatfully acknowledged.

\section{References}

1. J. G. BAKUCKAS and J. AWERBUCH, in "ISTFA Advanced Materials Symposium" (ASM International, Metals Park, OH, 1987) p. 33.

2. T. OHIRA, T. KISHI and R. HORIUCHI, in "Proceedings of the 5th International AE Symposium" (The Japanese Society for Non-Destructive Inspection, Tokyo, 1980) p. 137.

3. S. YUYAMA, T. KISHI, Y. HISAMATSU and T. KAKIMI, in "Progress in Acoustic Emission", Proceedings of the 6th International AE Symposium, edited by M. Onoe, K. Yamaguchi and T. Kishi (The Japanese Society for Non-Destructive Inspection, Susono, Japan, 1982) p. 126.

4. G. CHANCY, B. PIGE and H. PAQUETON, in "Titanium, Science and Technology", Proceedings of the 5th International
Conference on Titanium, edited by G. Lutjering, U. Zwicker and W. Bunk (Deutsche Gesellschaft Fur Metallkunde, Oberursel, FRG. 1985) p. 1891.

5. M. A. FRIESEL and S. H. CARPENTER, Met. Trans. 15A (1984) 1849.

6. Idem., Mater. Sci. Engng 68 (1984) 107.

7. J. R. KEN NEDY, Script Metall. 16 (1982) 525.

8. H. TANAKA and R. HORIUCHI, ibid. 9 (1975) 777.

9. T. KISHI, H. S. PARK, R. HORIUCHI, T. KAKIMI, M. NAKANOSE and T. TANABE, in "Titanium ' 80 Science and Technology", Proceedings of the 4th International Conference on Titanium, edited by $\mathrm{H}$. Kimura and $\mathrm{O}$. Izumi (The Metallurgical Society of AIME, Warrendale, PA, 1980) p. 1709.

10. T. KISHI, T. OHIRA and H. OHYAMA, in "Titanium Science and Technology", Proceedings of the 5th International Conference on Titanium, edited by G. Lutjering, U. Zwicker and W. Bunk (Deutsche Gesellschaft Fur Metallkunde, Oberursel, FRG, 1985) p. 2039.

11. C. E. HARTBOWER, C. F. MORAIS, W. G. REUTER and P. P. CRIMMINS, Engng Fract. Mech. 5 (1973) 765.

12. O. A. SHINAISHIN, M. S. DARLOW and S. J. ACQUAVIVA, Mater. Eval. 34 (1976) 137.

13. D. H. KOHN, P. DUCHEYNE and J. AWERBUCH, J. Mater. Sci. (1992) in press.

14. ASTM standard F136-84, in "Annual Book of ASTM Standards, Vol 13.01 "Medical Devices" (ASTM, Philadelphia, 1987) p. 28.

15. G. LUTJERING, A. GYSLER and L. WAGNER, in "Titanium Science Technology and Applications", Proceedings of the 6th World Conference on Titanium, edited by P. Lacombe, R. Tricot, and G. Beranger (Les Editions de Physique, Paris, 1989) p. 2.

16. L. WAGNER and G. LUTJERING, ibid.

17. D. BROEK, "Elementary Engineering Fracture Mechanics", (Martinus Nijhoff, The Hague, 1984).

Received 21 November 1990

and accepted 15 January 1991 